This item was submitted to Loughborough's Research Repository by the author.

Items in Figshare are protected by copyright, with all rights reserved, unless otherwise indicated.

\title{
Modifying landing mat material properties may decrease peak contact forces but increase forefoot forces in gymnastics landings
}

PLEASE CITE THE PUBLISHED VERSION

http://dx.doi.org/10.1080/14763141.2010.524244

\section{PUBLISHER}

Routledge (@ Taylor and Francis)

VERSION

AM (Accepted Manuscript)

\section{LICENCE}

CC BY-NC-ND 4.0

\section{REPOSITORY RECORD}

Mills, Chris, Maurice R. Yeadon, and Matthew T.G. Pain. 2019. "Modifying Landing Mat Material Properties May Decrease Peak Contact Forces but Increase Forefoot Forces in Gymnastics Landings". figshare. https://hdl.handle.net/2134/8153. 
This item was submitted to Loughborough's Institutional Repository (https://dspace.lboro.ac.uk/) by the author and is made available under the following Creative Commons Licence conditions.

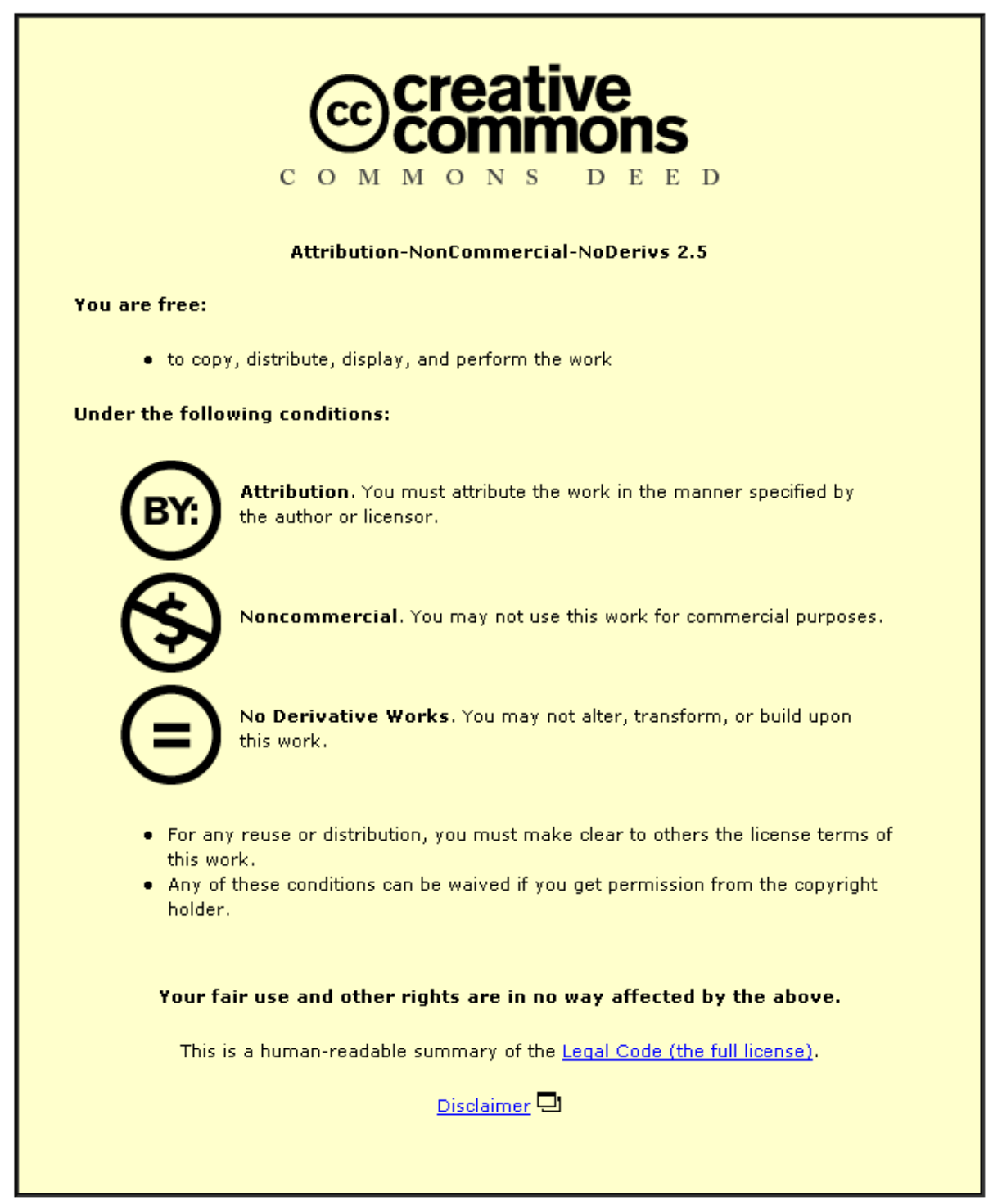

For the full text of this licence, please go to: http://creativecommons.org/licenses/by-nc-nd/2.5/ 
Sports Biomechanics 2010, 9, 153-164

\title{
Modifying landing mat material properties may decrease peak contact forces but increase forefoot forces in gymnastics landings
}

\author{
Chris Mills ${ }^{1}$, Maurice R. Yeadon ${ }^{2}$ and Matthew T.G. Pain ${ }^{2}$ \\ ${ }^{1}$ Sport and Exercise Science Department, University of Portsmouth, Portsmouth, UK \\ ${ }^{2}$ School of Sport, Exercise and Health Sciences, Loughborough University, Loughborough, UK
}

\begin{abstract}
This study investigated how changes in the material properties of a landing mat could minimise ground reaction forces (GRF) and internal loading on a gymnast during landing. A multi-layer model of a gymnastics competition landing mat and a subject-specific seven link wobbling mass model of a gymnast were developed to address this aim. Landing mat properties (stiffness and damping) were optimised using a Simplex algorithm to minimise GRF and internal loading. The optimisation of the landing mat parameters was characterised by minimal changes to the mat's stiffness $(<0.5 \%)$ but increased damping $(272 \%)$ compared to the competition landing mat. Changes to the landing mat resulted in reduced peak vertical and horizontal GRF and reduced bone bending moments in the shank and thigh compared to a matching simulation. Peak bone bending moments within the thigh and shank were reduced by $6 \%$ from $321.5 \mathrm{Nm}$ to $302.5 \mathrm{Nm}$ and GRF by $12 \%$ from $8626 \mathrm{~N}$ to $7552 \mathrm{~N}$ when compared to a matching simulation. The reduction in these forces may help to reduce the risk of bone fracture injury associated with a single landing and reduce the risk of a chronic injury such as a stress fracture.
\end{abstract}

Keywords : forces, impact, injury, model, simulation

\section{Introduction}

Many sporting activities involve a landing phase, whether repeated landings of each foot during running or a single double-footed landing from a vault in Artistic Gymnastics. It has been reported that up to $82 \%$ of injuries in gymnastics are acute and that the lower extremity was the most injured, comprising $54 \%$ to $70 \%$ of all injuries (Harringe et al., 2006; Jensen, 1998; Meeusen and Borms, 1992). Several factors may contribute to the frequency and severity of injuries during landing, including body position at the instant of touchdown, landing technique and the landing surface (Dufek and Bates, 1991; McNitt-Gray et al, 1993; McNitt-Gray, 2000; Mills et al., 2009).

In Artistic Gymnastics the landing mat is standardised by the international governing body (Federation Internationale Gymnastique, F.I.G.) and their standards are based upon the need to establish uniformity of the equipment used during competition rather than purely musculo-skeletal requirements (McNitt-Gray, 2000). The landing mat in Artistic Gymnastics is standardised using a series of rigid body drop tests. Peak force, deformation and rebound height are used to assess the cushioning properties of the landing mats and the results must adhere to the published standards in order to be used for international competitions (F.I.G., 2000). Additionally, mats are not re-tested periodically during their use and repeated impacts can decrease their cushioning properties (Wieners et al., 1995) possibly resulting in mats that fall outside the limits specified by the F.I.G. Due to the current standards tests using rigid 
impactors, using a model of human landing would be an improvement and provide additional insight into the loading on a gymnast resulting from mat material changes.

Judging criteria for landings in gymnastics stipulate that gymnasts must land with a single placement of the feet (no additional steps) with the centre of mass over the base of support. Any steps or unsteadiness, excessive arm swinging or loss of balance can result in a score deduction between 0.1 and 0.5 (F.I.G., 2009). The high incidence of injury associated with landing (Snook, 1979; Andrish, 1985; McAuley et al., 1987; Meeusen and Borms, 1992; Jensen, 1998; Daly et al., 2001; Kirialanis et al., 2003; Marshall et al., 2007) and the landing errors observed by McNitt-Gray (2000) indicate that gymnasts are having difficulty satisfying both safety and performance objectives. Marshall et al. (2007) also recommended that equipment manufacturers should be encouraged to re-evaluate the design of the landing mats to allow for better absorption of forces due to the injury rates associated with landing in gymnastics. An investigation of how changes to landing mat material properties could reduce these forces would help to enhance the knowledge in this area and contribute to the development of safer landing mats.

The interaction between a gymnast and the equipment is critical for maximising performance. An increase in skill complexity (maximising performance) can be associated with an increase in the height required to complete the skill (Takei, 1988; King et al., 1999). Ground impact forces generally increase with greater drop height (Dufek and Bates, 1991; McNitt-Gray et al., 1993) and greater impact forces and higher loading rates suggest a greater injury risk to the lower extremity (O'Leary et al., 2008). These injuries could be either acute or chronic since exposure to high levels of loading which are sub-injurious may still lead to injury (Dixon et al., 2003). Landing mat characteristics have been identified as key factors in the dissipation of forces during landing (Dufek and Bates, 1991; McNitt-Gray et al., 1993). Previous research has shown that humans usually use more joint flexion as the rigidity of the surface increases (Nigg and Yeadon, 1987; McNitt-Gray et al., 1994; Yeow et al., 2009, Yeow et al., 2010). However, there is also evidence that gymnasts regulate their landing forces via the amount of joint flexion used irrespective of surface compliance (McNitt-Grey et al., 1993). Yet it has also been shown that knee joint load increases as surface hardness increases and it has been suggested that this increased load may be a possible cause of injury when landing on harder surfaces (Dufek and Bates, 1991; Seegmiller and McCaw, 2003).

From practice to competition gymnasts may use progressively stiffer mats. Normally humans increase joint flexion to accommodate the increase in mat stiffness and ensure internal forces are stabilised. In gymnastics, however, deductions for landing may encourage the gymnast to use less joint flexion and risk of injury may increase as a result. In a simple skill less joint flexion is required to execute the preferred landing technique on a mat and so there is a greater range of motion available to the gymnast to accommodate unexpected events (McNitt-Gray, 1993). In a complex skill the gymnast will land with greater knee and hip flexion and will therefore have less range of motion available to accommodate landing errors (McNitt-Gray et al., 1993). This may lead to a greater chance of injury especially on a thinner and stiffer landing mat (Devita and Skelly, 1992).

Although the F.I.G. landing mat is currently standardised, a better or 'safer' landing mat may be possible that could reduce injury risk whilst allowing the gymnast to maintain a landing technique that minimises landing deductions. A computer simulation model of a gymnast and landing mat could be a useful tool when investigating how the material properties of landing mats could be modified to decrease the gymnast's musculoskeletal loading whilst maintaining the same landing technique ensuring minimal landing deductions. The level at which musculoskeletal loading could cause injury will not be specified as, apart from rare individual estimates (e.g. Zenicke et al., 1977), there are no known in vivo values 
for subject-specific injuries. In vitro fracture threshold values of near $400 \mathrm{~N} . \mathrm{m}$ have been reported for femoral samples (Marten et al., 1986). However, multiple muscles and the passive tissues surrounding the bone introduce many unknowns into in vivo bone loading, therefore only the generic risk of injury from increased loading can be assessed rather than the probability of injury.

This study investigated how changes in the material properties of a landing mat could minimise internal loading and external forces experienced by the gymnast during landing. The use of a forward dynamics approach to address the research issue provides an insight into mat properties which are otherwise difficult to examine experimentally due to potential injury to the gymnast.

\section{Methods}

A planar forward dynamics seven link muscle-driven wobbling mass model representing the gymnast (height $1.77 \mathrm{~m}$, mass $75 \mathrm{~kg}$ ) was constructed in visualNastran 4D (VN4D) to simulate the first $100 \mathrm{~ms}$ of landing on a competition gymnastics mat as detailed in Mills et al. (2008). The gymnast model (Figure 1) comprised a head + trunk, upper arm, lower arm + hand, thigh, shank and a two-segment foot; pin joints connected adjacent links at their ends and along the longitudinal axis of the segment (Mills et al., 2008). Internal loading was assessed using the shank and thigh links. Each segment was made of two parts connected by a rigid joint so that moments could be calculated in the middle of the link. Using a twopart link to calculate bending moment is a simple method to determine the magnitude of the loading on the skeletal structure and can be related to the fracture failure of bones from invitro tests (Martens et al., 1986). However, live bone is normally stronger than in vitro bone specimens and also has the advantage that muscle and other soft tissue surround it. These tissues can reduce the bending moments on the bone but may increase axial forces (Bertram and Biewener, 1988) although this was not accounted for in the model.

Personalised segmental inertia parameters for the gymnast were calculated using the inertia model of Yeadon (1990) and were then divided into rigid and wobbling mass inertial parameters based upon Pain and Challis (2006). A 'lumped' muscle model with subjectspecific strength parameters represented all the muscles responsible for flexion at a particular joint and a similar muscle model was used for extension (Mills et al., 2008). This allowed the linear actuator to operate with a moment arm scaled to the subject from the middle of the ranges found in the work of Duda et al. (1996) and Jacobs et al. (1996).

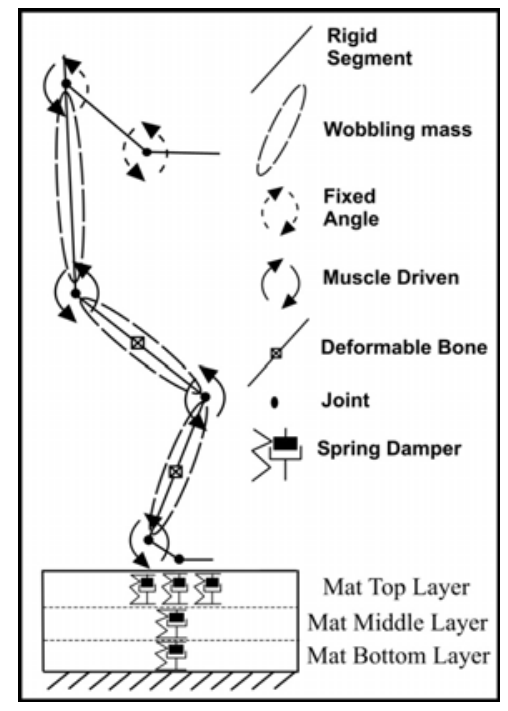

Fig. 1. Gymnast and landing mat model construction. 
Material properties of the competition landing mat were based upon rigid body impact tests performed independently (Pain et al., 2005). Results in Pain et al. (2005) showed that the effective mass being accelerated, for the size of mat used in the study, corresponded to a force difference between the top and bottom of the mat that ranged from $480 \mathrm{~N}$ to $930 \mathrm{~N}$, accounting for up to $12 \%$ of the peak force during the trials (Pain et al., 2005). The landing mat model used in this study was based upon the same multi-layer spring-damper design and evaluated using independent impact (Mills et al., 2006). This landing mat model included sufficient complexity to allow the modification of the stiffness and damping parameters for each of the layers of the mat and the effective mat mass of each layer, although the latter were kept constant during this study. In order for the whole mat model to behave in a physically relevant manner it is important to have each layer of the mat modelled with the appropriate effective mass so that changes at one interface or layer propagate through correctly to the other layers and interfaces. Simulation results showed the mat model could reproduce the key characteristics of the ground reaction force at impact (Mills et al., 2006). The mat model also included three points of contact at the foot to mat interface to allow forefoot landings in future applications (Figure 1). The spring-damper parameters from the matching simulation during independent impact tests (Mills et al., 2006) were used as the starting point for subsequent landing mat optimisations.

The gymnast-mat model has been evaluated using results from experimental forward and backward rotating somersault vaults (Mills et al., 2008). The use of different vaults helps to address the suitability of any mat modification to a range of landing techniques. In this process matching simulations of the real vaults were produced by minimising the kinematic and kinetic differences between the recorded performance and the model output as detailed in Mills et al. (2008). This was done to provide a base with which to compare the optimised simulations. The gymnast-mat model produced matching simulations of the landings with overall kinematic and kinetic errors of $10-15 \%$ (Mills et al., 2008). Initial conditions comprising limb and whole body positions, velocities and orientations, as well as muscle activation time histories for the model during optimisations were kept the same as in the matching simulations. Although the muscle activation patterns were not re-optimised for each change in mat properties, this model still represents a significant development in the understanding of loading on a gymnast over the previously used rigid body impact tests. The parameters for the competition-landing mat were used as a starting point for the future optimisations. Since the muscle activation histories remained unchanged any decrease in external GRF may also result in decreased internal loading (bone bending moments). The magnitude of the bone bending moments within the lower extremities was used as a means of assessing the risk of injury during the landings. The gymnast-mat model was not designed to specify what injuries may occur but instead used to determine whether internal forces increase or decrease. If internal forces in the model decrease relative to the matching simulation, this may suggest a reduced injury risk to the gymnast in general.

The Simplex optimisation algorithm was used to vary the landing mat spring parameters and minimise the objective function $S_{1}=(F z+F y)+$ penalties, where $\mathrm{Fz}=$ peak VGRF (vertical ground reaction force) and Fy = peak HGRF (horizontal ground reaction force). Ideally the foot/mat forces should be minimised but serious problems due to the multipoint foot contacts and the limitations of the visualNastran modelling environment were encountered. As the number of foot contacts changed during the simulation the software tended to jump between solutions for total foot/mat force resulting in huge oscillations and an unrealistic solution. A simplified model with a single point of contact was used to look at the difference between minimising the foot/mat forces and the VGRF. The foot/mat force when minimising foot/mat force was $2669 \mathrm{~N}$ and the foot/mat force when minimising the VGRF 
was $2680 \mathrm{~N}$, less than 1\% difference. However, without multiple foot contacts a successful landing was not possible.

Constraints were placed upon the simulation during the optimisations to minimise the computational time, ensure landing technique did not incur deductions as defined by F.I.G. (Mills et al., 2009), and ensure the landing mat behaved within realistic limits. Landing mat constraints included ensuring the mat did not become excessively compressed and 'bottom out'. Each constraint was written as a penalty and added to the objective function score if the constraint was violated.

The upper and lower bounds for the stiffness and damping of each layer were set at \pm 20 $\%$ of the competition landing mat parameters presented in Table 1 . These were chosen to ensure the optimised landing mat parameters were within the construction capabilities of the manufacturer and close to the current F.I.G. landing mat. An additional bound of $\pm 1000 \%$ to maximise the search space was also set for a final optimisation to investigate if loading on the gymnast could be reduced further if manufacturing capabilities would allow.

Table 1. Matching simulation and optimised landing mat parameters for the forward (FR) and backward (BR) rotating vaults

\begin{tabular}{cccccc}
\hline Parameter & & $\begin{array}{c}\text { Matching } \\
\text { Simulation } \\
\text { Landing Mat }\end{array}$ & $\begin{array}{c}\text { Optimised } \\
\text { Landing } \\
\text { Mat (FR) }\end{array}$ & $\begin{array}{c}\text { Optimised } \\
\text { Landing Mat } \\
\text { (BR) }\end{array}$ & $\begin{array}{c}\text { Optimised } \\
\text { Landing } \\
\text { Mat (FR)** }\end{array}$ \\
\hline $\begin{array}{c}\text { Score } \\
\text { Penalties }\end{array}$ & & - & $8494 \mathrm{~N}$ & $8020 \mathrm{~N}$ & $7559 \mathrm{~N}$ \\
\hline Top layer stiffness & $(\mathrm{N} / \mathrm{m})$ & 61080 & 61197 & 61089 & 61063 \\
Middle layer stiffness & $(\mathrm{N} / \mathrm{m})$ & 57530 & 57591 & 57534 & 57529 \\
Bottom layer stiffness & $(\mathrm{N} / \mathrm{m})$ & 928120 & 928340 & 928210 & 928215 \\
Top layer damping & $(\mathrm{Ns} / \mathrm{m})$ & 350 & $420 *$ & $420 *$ & 940 \\
Middle layer damping & $(\mathrm{Ns} / \mathrm{m})$ & 430 & $516 *$ & 395 & 916 \\
Bottom layer damping & $(\mathrm{Ns} / \mathrm{m})$ & 290 & $348 *$ & $348 *$ & 1079 \\
\hline
\end{tabular}

$*$ = upper bound (20\%) reached

** = increased damping upper bound (1000\%)

\section{Results}

The optimisation of the six landing mat parameters ( $\pm 20 \%$ bounds) for the forward and backward rotating vault resulted in the peak VGRF and HGRF being reduced, whilst the gymnast model maintained an acceptable landing technique according to the F.I.G. criteria. The optimised mat reduced the VGRF by $84 \mathrm{~N}$ and the HGRF by $48 \mathrm{~N}$ during the forward rotating vault (Figure 2), when compared to a matching simulation (Mills et al., 2008). The VGRF was also reduced by $85 \mathrm{~N}$ and the HGRF by $31 \mathrm{~N}$ in the backward rotating vault (Figure 3), when compared to a matching simulation (Mills et al., 2008). The reduction in peak GRF was accompanied by a decrease in peak shank and thigh bone bending moments (1 $\%)$ in both the forward and backward rotating vaults compared to the matching simulation. 


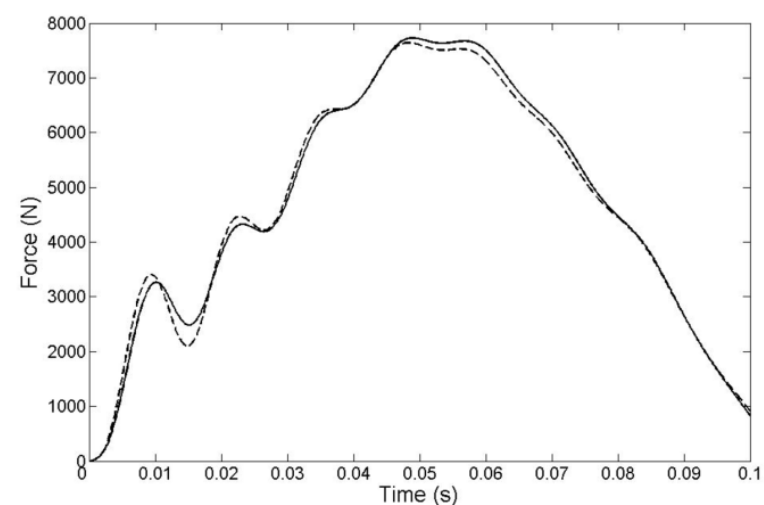

(a)

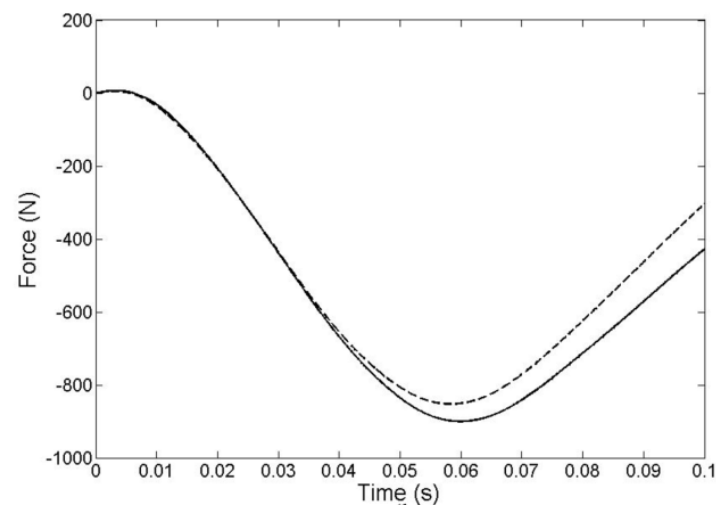

(b)

Fig. 2. VGRF and HGRF following an optimisation of landing mat properties for the forward rotating vault (a) VGRF (b) HGRF during the $\pm 20 \%$ stiffness and damping bound condition. (Solid line = matching simulation, dashed line $=$ optimised simulation).

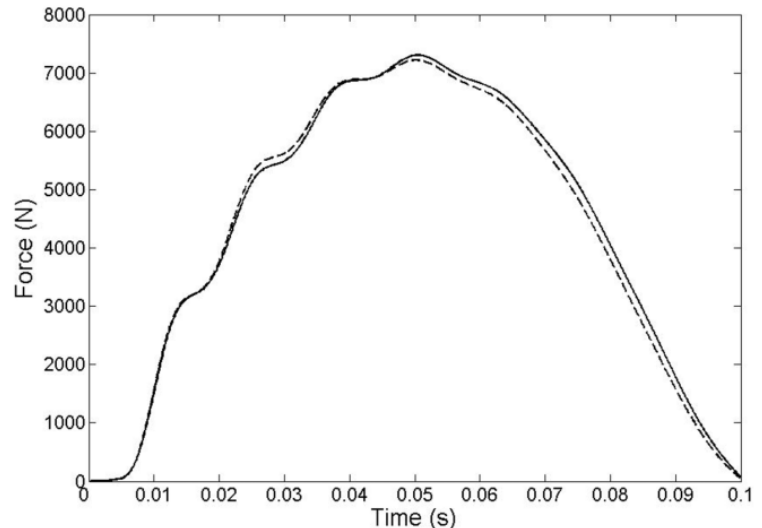

(a)

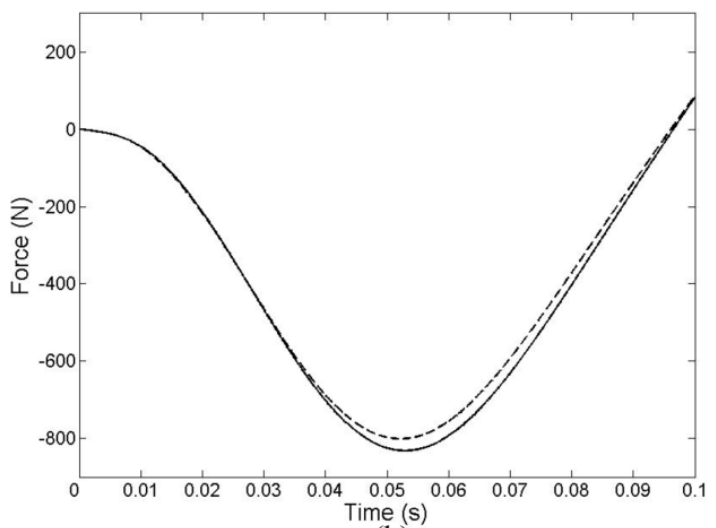

(b)

Fig. 3. VGRF and HGRF following an optimisation of landing mat properties for the backward rotating vault (a) VGRF (b) HGRF during the $\pm 20 \%$ stiffness and damping bound condition. (Solid line = matching simulation, dashed line $=$ optimised simulation $)$.

The optimisation of the landing mat material properties was characterised by minimal changes to the stiffness values for each layer $(<0.5 \%)$ compared to the matching simulation, although the damping parameters reached the upper bounds of $20 \%$ for each layer of the mat (Table 1). The $20 \%$ increase in damping within the optimised landing mat also caused the initial forefoot force to increase by $10 \%$ when compared to the matching simulation (Fig. 4).

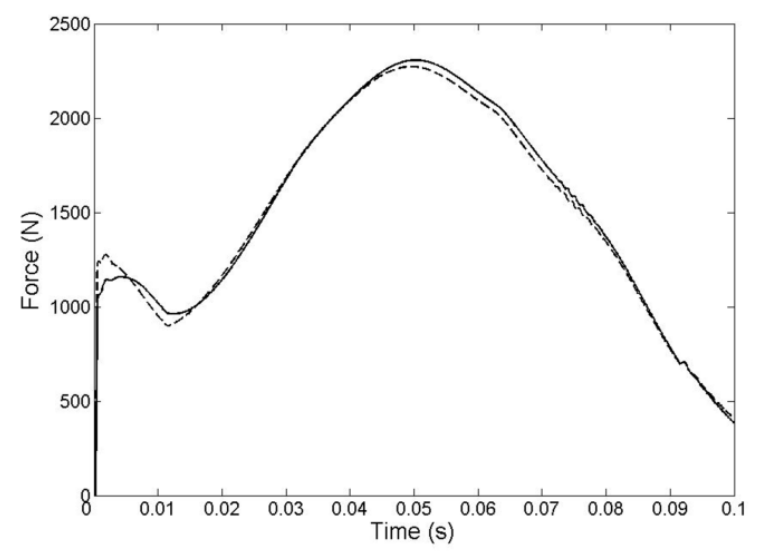

Fig. 4. Example of the increased initial forefoot force during a forward rotating vault with the $\pm 20 \%$ stiffness and damping bound condition. (Solid line $=$ matching simulation, dashed line $=$ optimised simulation). 
A further increase in the damping bound (from $\pm 20 \%$ to $\pm 1000 \%$ ) during an additional optimisation of the landing mat properties was characterised by a minimal change in mat stiffness $(<0.5 \%$ ) but increased damping (up to $272 \%$ ) at each layer (Table 1 ). The further increase in mat damping during this additional optimisation resulted in a greater decrease in both the peak VGRF and HGRF by $10 \%$ and $36 \%$ respectively (Figure 5) and a 5\% and $13 \%$ decrease in the vertical and horizontal average loading rates respectively (Table 2) compared to the matching simulation.

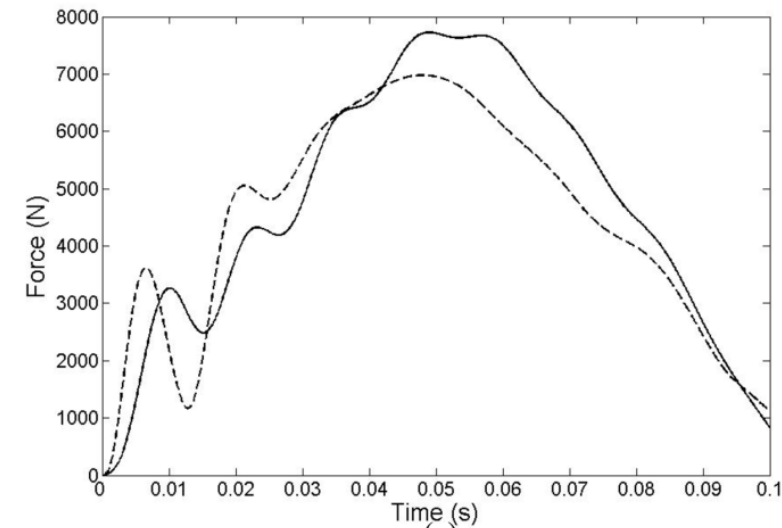

(a)

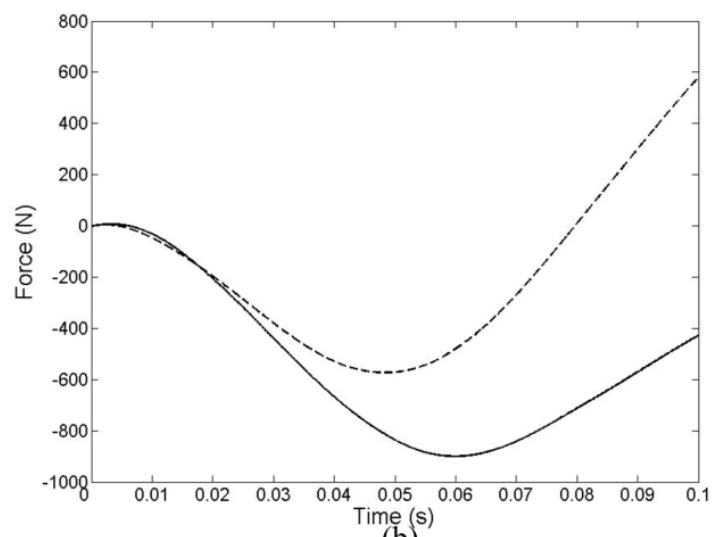

(b)

Fig. 5. VGRF and HGRF following an optimisation of landing mat properties for the forward rotating vault with increased ( $\pm 1000 \%$ ) damping bound (a) VGRF (b) HGRF. (Solid line = matching simulation, dashed line $=$ optimised simulation).

Table 2. Average loading rates (peak VGRF / time to peak) for the matching simulation and optimised landing mat parameters for the forward (FR) and backward (BR) rotating vaults.

\begin{tabular}{|c|c|c|c|c|c|}
\hline Parameter & $\begin{array}{c}\text { Matching } \\
\text { Simulation } \\
\text { Landing Mat } \\
\text { (FR) }\end{array}$ & $\begin{array}{c}\text { Matching } \\
\text { Simulation } \\
\text { Landing Mat } \\
\text { (BR) }\end{array}$ & $\begin{array}{c}\text { Optimised } \\
\text { Landing } \\
\text { Mat } \\
\text { (FR) }\end{array}$ & $\begin{array}{c}\text { Optimised } \\
\text { Landing } \\
\text { Mat } \\
\text { (BR)* }\end{array}$ & $\begin{array}{c}\text { Optimised } \\
\text { Landing } \\
\text { Mat } \\
\text { (FR)** }\end{array}$ \\
\hline $\begin{array}{l}\text { Average VGRF loading } \\
\text { rate }(\mathrm{N} / \mathrm{ms})\end{array}$ & 158 & 146 & 159 & 144 & 145 \\
\hline $\begin{array}{l}\text { Average HGRF loading } \\
\text { rate }(\mathrm{N} / \mathrm{ms})\end{array}$ & 15 & 16 & 15 & 15 & 12 \\
\hline $\begin{array}{l}\text { Average shank bone } \\
\text { loading rate }(\mathrm{Nm} / \mathrm{ms})\end{array}$ & 6 & 5 & 6 & 5 & 7 \\
\hline $\begin{array}{l}\text { Average thigh bone } \\
\text { loading rate }(\mathrm{Nm} / \mathrm{ms})\end{array}$ & 4 & 4 & 4 & 4 & 3 \\
\hline
\end{tabular}

$*$ = upper bound $(20 \%)$

** = increased damping upper bound (1000 \%)

The reduction in peak GRF during the $\pm 1000 \%$ bound optimisation also resulted in a decrease in peak shank and thigh bone bending moments. The peak shank bone moment decreased from 377 N.m to 356 N.m and the peak thigh bone moment decreased from 266 N.m to 249 N.m (Figure 6) compared to the matching simulation. The average loading rates increased by $17 \%$ at the shank but decreased by $25 \%$ at the thigh (Table 2) compared to the matching simulation. However, the increased bounds ( $\pm 1000 \%)$ optimisation caused a higher initial forefoot force (by $65 \%$ ) for the $272 \%$ increase in mat damping (Figure 7) compared to the matching simulation. 


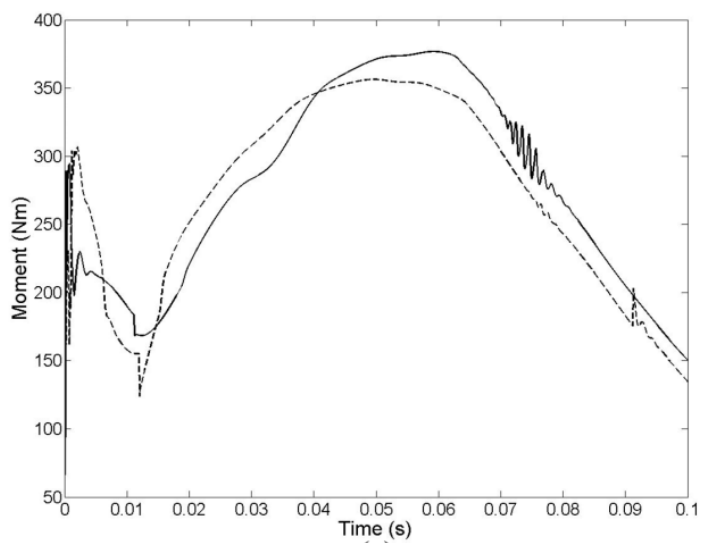

(a)



(b)

Fig. 6. Lower extremity bone bending moments during the forward rotating vault with increased $( \pm 1000 \%)$ damping bound (a) shank, (b) thigh. (Solid line = matching simulation, dashed line = optimised simulation). The latter vibration is due to high frequency simulation artefact from the muscle model.

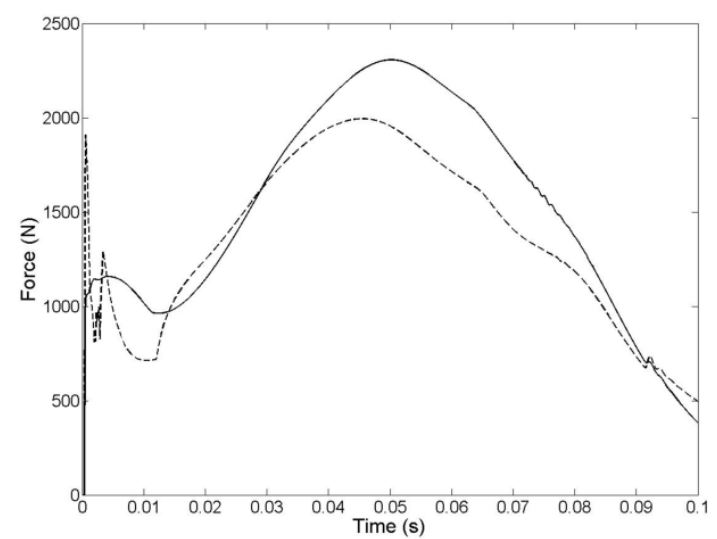

Fig. 7. Example of the further increase in initial forefoot force during a forward rotating vault with increased $( \pm 1000 \%)$ damping bound. (Solid line $=$ matching simulation, dashed line $=$ optimised simulation $)$.

\section{Discussion and Implications}

The aim of this study was to determine the material properties of a landing mat that could minimise ground reaction forces (GRF) and internal loading on a gymnast during landing. The results using the three layer mat model have shown that optimising the landing mat properties to minimise the peak GRF resulted in decreased peak VGRF, HGRF and average loading rates when compared to a matching simulation for both the forward and backward rotating vaults. The optimisation of the landing mat parameters was characterised by minimal changes to the landing mat's stiffness $(<0.5 \%)$ but increased damping (20\%). The upper bound of $+20 \%$ (20\% more damping than in the F.I.G. mat) was reached during the optimisation of both the forward and backward rotating vaults. A more damped landing mat (272\%) decreased the peak external forces and internal loading experienced during landing for the subject in this study.

The most significant finding of this study was that a minimal change in mat stiffness and a modification to the damping coefficient of the competition landing mat can reduce the GRF, average loading rates and internal loading on the gymnast during landing, whilst maintaining an acceptable landing technique. Such a reduction in forces on and inside the musculo-skeletal system could help to reduce the injury risk associated with landing (McNittGray et al., 2000). This was further supported by Marti et al. (1988) and Beck (1998) who 
stated that the main factor associated with bone stress injuries was repeated mechanical loading and that the amount of load seemed to correlate directly with the extent of injury. Additionally the use of cushioned insoles during running have been shown to reduce ground reaction forces by up to $6.8 \%$ and loading rates by $8.3 \%$ (O’Leary et al., 2008) and could potentially reduce overuse injuries (Dixon et al., 2003). In comparison, in the present study the GRF was reduced by $22.9 \%$, and the loading rate by $9 \%$.

Increasing the amount of damping in the landing mat was associated with a problem related to the forefoot force during initial touchdown. More damping in the mat increased the initial force on the forefoot and this may feel uncomfortable to a gymnast even though the peak VGRF acting on the model was reduced. The gymnast in this study had reported in the past a pain in the feet during 'stiffer' landings. Increasing the upper bound in the optimisation to $1000 \%$ that of the matching simulation (F.I.G. mat) allowed more damping to be selected for the optimised solution. The extra damping (approximately 2.7 times more than the F.I.G. mat) in the landing mat, which could possibly be implemented using Confor ${ }^{\circledR}$ Foam, resulted in further reductions in peak VGRF and HGRF combined with reduced peak shank and thigh bone bending moments. However, the initial forefoot force was increased from $1158 \mathrm{~N}$ in the matching simulation to $1910 \mathrm{~N}$ in the optimised mat for the forward rotating vault. This again may make the landing feel uncomfortable to a gymnast; however the risk of injury due to high external and internal forces may be reduced. This is an interesting finding and further investigation could aim to understand the influence of increased mat damping on localised foot injuries.

The role of the ankle and foot to attenuate forces during landings has been shown to be of great importance (Kovacs et al., 1997). In the future it would be interesting to investigate the role of the forefoot in landings and the feel / perception of discomfort versus actual increased injury risk from localised forces within the foot. To help reduce this problem the landing mat with increased damping could be combined with a softer thin non-viscous additional top layer, which could reduce the initial impact peak on contact. Recent developments in gymnastics regulations have allowed the use of an optional 'throw in' mat. This thin soft mat may help to reduce the initial impact force between the foot and the mat's surface. Future research could also aim to investigate the use of a thinner softer additional mat and its effectiveness in reducing the initial impact peak whilst maintaining the levels of increased damping in the main landing mat with the purpose of providing useful information on mat construction. Future research could also incorporate inertial modifications to the subject to determine whether similar material properties could also benefit the lighter, female gymnast and landing technique changes to reflect the different strategies used by gymnasts.

It is noted that the optimum for the three layer mat model construction may not be the optimum mat construction. With greater variations in mat properties than those used in the evaluations the differences between minimising foot / mat forces and GRF may increase. It is possible that varying the mass of the layers, although this is harder to do independently of spring-damper properties, or moving to a two layer mat or a single layer non-linear mat may produce better results. On the other hand these simpler models have been shown to struggle to reproduce the key deformation characteristics during impact (Mills et al., 2006). The mat layers are also limited as surface deformation that starts at the point of contact moving out towards the outer edges of the mat (Pain et al., 2005) could not be reproduced in the mat model since only the entire mat layer could deform. However, simulated mat deformations were realistic at 10-11 cm which was comparable to the range of 9-12 cm reported in Mills et al., (2006). An improvement would be to use finite element modelling to map the mat layers although when this approach was attempted the simulation time increased to a point where 
any optimisation was impractical. The three layer approach adopted in this study reproduced the key mat deformations characteristics and allowed many simulations to be run.

The increased damping in the optimised mat, making it more viscoelastic, may also alter the physical recovery of the mat as it relies on gaining energy from the environment in order to re-establish its original structure; this delay in the mat's ability to restore to its original shape may cause a problem with the stability and recovery of balance of the gymnast during completion of the land and stop skill. This may not be a major problem for the gymnast since the landing strategy could be modified to accommodate the delay ensuring successful retention of balance. However, future research incorporating the material mat changes and landing strategy adjustments could investigate this interaction.

The gymnast model does not account for the three-dimensional components of multiple muscle insertions and geometry, which will change the internal bone loading patterns. In reality the more complex structure of human legs has a greater potential for reducing loading but it also introduces a larger set of variables and parameters from a modelling perspective. As a consequence the internal loading in the model is likely to be higher than in actual human bone in situ. In vitro femoral samples have been reported to fail near 400 N.m (Marten et al., 1986) but as there are no experimental measures of bone bending moments during vault landings a true comparison is problematic. Although the model may over-estimate the actual loading on the bone the model is still able to determine the comparative effects of different mat material properties on internal loading and could help to reduce landing related injuries.

The optimal landing mat solutions found here are likely to be local minima, near to the original F.I.G. competition mat and although this may be a shortcoming of the study in methodological terms it does not affect the major finding. Local solutions may also be more useful from an applied point of view as a true global solution may require very different manufacturing processes to construct the landing mat. The local minimum solution means that through minor stiffness and larger damping changes to the material properties of the mat would result in reduced internal loading, whilst keeping other characteristics such as the multi-layer design, effective mass and thickness the same as the current F.I.G. landing mat. The reduction in the internal loading and external forces may help to reduce the risk of bone fracture injury associated with a single landing and reduce the risk of a chronic injury such as a stress fracture.

\section{Conclusion}

It can be concluded that the stiffness of the current competition landing mat is optimum and a $20 \%$ change in landing mat damping results in only a small reduction in internal forces within the gymnast, however a large increase in damping (272\%) would be of benefit and possibly reduce injury risk associated with vault landing in gymnastics. It must also be noted that increased mat damping may be associated with increased forefoot loading; further investigation should aim to understand its influence on localised injuries. These subject specific findings provide a starting point for future investigation and may have implications for future mat construction, possible modifications to gymnastics equipment regulations and the possibility of reducing the injury risk of gymnasts. 


\section{References}

Beck, B. (1998). Tibial stress injuries: an aetiological review for the purposes of guiding management. Sports Medicine, 26, 265-279.

Bertram, J. \& Biewener, A. (1988). Bone Curvature: Sacrificing strength for load predictability? Journal of Theoretical Biology, 131, 75-92.

Daly, R., Bass, S. \& Finch, C. (2001). Balancing the risk of injury to gymnasts: how effective are the counter measures? British Journal of Sports Medicine, 35, 8-19.

Devita, P. \& Skelly, W. (1992). Effect of landing stiffness on joint kinetics and energetics in the lower extremity. Medicine and Science in Sports and Exercise, 24(1), 108-115.

Dixon, S.J., Waterworth, C., Smith, C. \& House, C. (2003). Biomechanical analysis of running in military boots with new and degraded insoles. Medicine and Science in Sports and Exercise, 35, 472-479.

Duda, G., Brand, D., Freitag, S., Lierse, W. \& Schneider, E. (1996). Variability of femoral muscle attachments. Journal of Biomechanics, 29, 1185-1190.

Dufek, J. T. \& Bates, B. T. (1991). Biomechanical factors associated with injury during landing in jump sports. Sports Medicine, 12, 326-337.

Federation Internationale de Gymnastique - F.I.G. (2000). Apparatus Norms. F.I.G., Switzerland.

Federation Internationale de Gymnastique - F.I.G. (2009). Code of Points - men's artistic gymnastics. F.I.G., Switzerland.

Harringe, M., Renstrom, P., Werner, S. (2006). Injury incidence, mechanism and diagnosis in top-level teamgym: a prospective study conducted over one season. Scandinavian Journal of Medicine and Science in Sports, 1-5.

Jacobs, R., Bobbert, M. \& van Ingen Schenau, G. (1996). Mechanical output from individual muscles during explosive leg extensions: the role of biarticular muscles. Journal of Biomechanics, 29, 513-523.

Jensen, J. E. (1998). Stress fracture in a world-class athlete: a case study. Medicine and Science in Sports and Exercise, 30, 783-787.

King, M. A., Yeadon, M. R., Kerwin, D. G., 1999. A two-segment simulation model of long horse vaulting. Journal of Sports Sciences 17, 313-324.

Kirialanis, P., Malliou, P., Beneka, A. \& Giannakopoulos, K. (2003). Occurrence of acute lower limb injuries in artistic gymnasts in relation to event and exercise phase. British Journal of Sports Medicine, 37, 137-139.

Kovacs, I., Tihanyi, J., DeVita, P., Racz, L., Barrier, J. \& Hortobagyi, T. (1997). Foot placement modifies kinematics and kinetics during drop jumping. Medicine \& Science in Sports \& Exercise, 31, 708-716.

Marshall, S., Covassin, T., Dick, R., Nassar, L. \& Agel, J. (2007). Descriptive epidemiology of collegiate women's gymnastics injuries: National collegiate athletic association injury surveillance system, 1988-1989 through 2003-2004. Journal of Athletic Training, 42, 234-240.

Martens, M., van Audekercke, R., de Meester, P. \& Mulier, J.C. (1986). Mechanical behaviour of femoral bones in bending loading. Journal of Biomechanics, 19, 443-54.

Marti, B., Vader, J.P. \& Minder, C.E. (1988). On the epidemiology of running injuires. The 1984 Berne Grand Prix study. American Journal of Sports Medicine, 16, 285-294. 
McNitt-Gray, J. L. (2000). Musculoskeletal Loading During Landing. In: Zatsiorsky, V. (Eds.), The Encyclopaedia of Sports Medicine: Biomechanics in Sports. IOC, Blackwell Science, pp.523-549.

McNitt-Gray, J. (1993). Kinetics of the lower extremities during drop landings from three heights. Journal of Biomechanics, 26, 1037-1046.

McNitt-Gray, J. L., Yokoi, T. \& Millward, C. (1993). Landing strategy adjustments made by female gymnasts in response to drop height and mat composition. Journal of Applied Biomechanics, 9, 173-190.

McNitt-Gray, J. L., Yokoi, T. \& Millward, C. (1994). Landing strategies used by gymnasts on different surfaces. Journal of Applied Biomechanics, 10, 237-252.

Meeusen, R. \& Borms, J. (1992). Gymnastic injuries. Sports Medicine, 13, 337-356.

Mills, C., Pain, M. T. G. \& Yeadon, M. R. (2006). Modelling a viscoelastic gymnastics landing mat during impact. Journal of Applied Biomechanics, 22, 103-111.

Mills, C., Pain, M. T.G. \& Yeadon, M. R. (2008). The influence of simulation model complexity on the estimation of internal loading in gymnastics. Journal of Biomechanics, 41, 620-628.

Mills, C., Pain, M. T.G. \& Yeadon, M. R. (2009). Reducing ground reaction forces in gymnastics' landings may increase internal loading. Journal of Biomechanics, 42, 671678.

Nigg, B. \& Yeadon, M. R. (1987). Biomechanical aspects of playing surfaces. Journal of Sports Sciences, 5, 117-145.

O’Leary, K., Vorpahl, K.A. \& Heiderscheit, B. (2008). Effect of cushioned insoles on impact forces during running. Journal of the American Podiatric Medical Association, 98, 3641.

Pain, M.T.G. \& Challis, J. H. (2006). The influence of soft tissue movement on ground reaction forces, joint torques and joint reaction forces in drop landings. Journal of Biomechanics, 39, 119-124.

Pain, M. T. G., Mills, C. \& Yeadon, M.R. (2005). Video analysis of the deformation and effective mass of gymnastics landing mats. Medicine and Science in Sports and Exercise, 37, 1754-1760.

Seegmiller, J. \& McCaw, S. (2003). Ground reaction forces among gymnasts and recreational athletes in drop landings. Journal of Athletic Training, 38, 311-314.

Takei, Y., 1988. Techniques used in performing handspring and salto forward tucked in gymnastics vaulting. International Journal of Sports Biomechanics, 4, 260-281.

Wieners, A., Michael, F., Stallkamp, K. \& Nicol, K. (1995). Decrease of cushioning properties of sportsmats. In: XVth Congress of the International Society of Biomechanics, Jyvaskyla, Finland.

Yeadon, M. R. (1990). The simulation of aerial movement - II. A mathematical inertia model of the human body. Journal of Biomechanics, 23, 67-74.

Yeow, C., Lee, P. \& Goh, J. (2009). Regression relationships of landing height with ground reaction forces, knee flexion angles, angular velocities and joint powers during doubleleg landing. The Knee, 16, 381-386.

Yeow, C., Lee, P. \& Goh, J. (2010). Sagittal knee joint kinematics and energetic in response to different landing heights and techniques. The Knee, 17, 127-131.

Zernicke, R. F., Garhammer, J. \& Jobe, F. W. (1977). Human patellar-tendon rupture. Journal of Bone and Joint Surgery, 59, 179-183. 